\title{
Optimization of sliding mode control with PID surface for robot manipulator by Evolutionary Algorithms
}

https://doi.org/10.1515/comp-2020-0144

Received Jul 15, 2019; accepted Apr 16, 2020

\begin{abstract}
In this paper, a sliding mode controller (SMC) with PID surface is designed for the trajectory tracking control of a robot manipulator using different optimization algorithms such as, Antlion Optimization Algorithm (ALO) Sine Cosine Algorithm (SCA) Grey Wolf Optimizer (GWO) and Whale Optimizer Algorithm (WOA). The aim of this work is to introduce a novel SMC-PID-ALO to control nonlinear systems, especially the position of two of the joints of a 2DOF robot manipulator. The basic idea is to determinate four optimal parameters ( $K_{p}, K_{i}, K_{d}$ and lamda) ensuring the best performance of a robot manipulator system, minimizing the integral time absolute error criterion (ITAE) and the integral time square error criterion (ISTE). The robot manipulator is modeled in Simulink and the control is implemented using the MATLAB environment. The obtained simulation results prove the robustness of ALO in comparison with other algorithms.
\end{abstract}

Keywords: Sliding mode control, Optimization algorithms, Robot manipulator

\section{Introduction}

In industrial applications, the use of robotic arms has increased significantly in recent years. Robot motion tracking controls, which requires high accuracy, stability and safety, is one of the primary challenging problems in this regard, largely due to it being highly coupled and nonlinear dynamic. In the presence of model uncertainties such as dynamic parameters (e.g. inertia and payload condi-

\footnotetext{
^Corresponding Author: Fatiha Loucif: Laboratoire d'Automatique et Informatique de Guelma, Université 8 Mai 1945, Guelma, Algeria; Email: loucif.fatiha@univ-guelma.dz Sihem Kechida: Laboratoire d'Automatique et Informatique de Guelma, Université 8 Mai 1945, Guelma, Algeria; Email: kechida.sihem@univ-guelma.dz
}

tions), dynamic effects (e.g. complex nonlinear frictions) and unmodeled dynamics, conventional controllers have many difficulties in treating these uncertainties. To overcome these drawbacks, we use the Sliding mode control (SMC) since it is one of the most robust approaches. Its most distinguished property lies in its insensitivity to dynamic uncertainties and external disturbances. Howeve, this approach exhibits high frequency oscillations called chattering when the system state reaches the sliding surface, which has negative effects on the actuators control and excite the undesirable unmodeled dynamics. Recently, proportional integral derivative sliding mode controllers (SMC-PIDs) have been examined as a powerful nonlinear controller[1-7]. An adaptive sliding mode control was treated in several papers [8-11]. The investigation of fuzzy logic with SMC was realized in many works [12-18]. In order to improve the performance of SMC control, GA was applied to determine once the optimal parameters of a conventional SMC controller [19-21] and once again to find the optimal rules and membership functions of the fuzzy sliding mode controller [22, 23]. Vijay and Jena designed a PSO based backstepping sliding mode controller and observer for robot manipulators [24]. The authors in [25, 26] used PSO to tune sliding surface parameters of SMC coupled with an artificial neuro fuzzy inference system (ANFIS) and to adjust the membership functions of Takagi Sugeno fuzzy SMC respectively. A new combination of a PSO algorithm with super twisting SMC for chattering elimination is proposed in [27]. Medjghou et al. have presented an optimized extended Kalman filter with integral type 2 fuzzy SMC using Biogeography-based optimization (BBO) [28]. Conventional SMC was optimized by colonial competitive algorithms in [29]. Oliveira et al. have proposed the use of GWO with a chaotic basis to tune robust higher order SMC for the position control of a robot manipulator [30]. More recently, Mokeddem and Draidi introduced the SMC-PID to control a second order linear system [31]. In their work, they proved that the optimization of controller parameters using ALO significantly outperforms GA and PSO algorithms. Loucif et al. have presented the optimiza-

○ Open Access. (C) 2020 F. Loucif and S. Kechida, published by De Gruyter. 
tion of PID controllers using WOA algorithms for the trajectory tracking control of 2DOF robot manipulators [32]. in this paper, a novel optimal PID Controller for the trajectory tracking control of a 2DOF Robotic Manipulator System is proposed which provides a new application to tune SMC with PID surface using ALO for the control of position of two joints. In order to show the performances of ALO, a comparative study based on the application of optimization algorithms was carried out. These algorithms are described in Section 3. While Section 2 defines the mathematical model of a robot manipulator. The principle of SMC and its application to control the robot manipulator are detailed in Section 4. Section 5 contains the simulation results and discussions followed by the conclusion.

\section{Dynamic model of robot manipulator}

By applying Lagrange's principle, the dynamic model of a two degrees of freedom (2DOF) robot manipulator is given by:

$$
\tau=M(q) \ddot{q}+C(q, \dot{q}) \dot{q}+G(q)+F(\dot{q})
$$

where, $q_{i}, \dot{q}_{i}$ and $\ddot{q}_{i}$ present the link position, velocity and acceleration vectors respectively. $M(q), H(q, \dot{q}), G(q)$ and $F(\dot{q})$ are respectively the matrix inertia, the Coriolis centripetal forces matrix, the gravity vector and the friction force vector.

$$
M(q)=\left[\begin{array}{ll}
M_{11} & M_{12} \\
M_{21} & M_{22}
\end{array}\right]
$$

with

$$
\begin{gathered}
M_{11}=m_{1} l_{1}^{2}+m_{2}\left(l_{1}^{2}+2 l_{1} l_{2} \cos \left(q_{2}\right)+l_{2}^{2}\right. \\
M_{12}=M_{21}=m_{2} l_{2}\left(l_{2}+l_{1} \cos \left(q_{2}\right)\right) \\
M_{22}=m_{2} l_{2}^{2} . \\
C(q)=\left[\begin{array}{ll}
C_{11} & C_{12} \\
C_{21} & C_{22}
\end{array}\right]
\end{gathered}
$$

\section{Evolutionary algorithms}

\subsection{SCA algorithm}

The SCA algorithm (Sine Cosine Algorithm) is a novel population-based optimization algorithm to solve optimization problems [33]. The SCA creates multiple initial random candidate solutions and forces them to fluctuate outward or toward the best solution using a mathematical model based on sine and cosine functions. To emphasize exploration and exploitation of the search space at different milestones of optimization, several random and adaptive variables are also integrated into this algorithm. In this 
algorithm, the following position updating equations are proposed for both phases:

$$
\begin{aligned}
& X_{i}^{t+1}=X_{i}^{t}+r_{1}{ }^{\star} \sin \left(r_{2}\right) \star\left|r_{3}{ }^{\star} p_{i}^{t}-X_{i}^{t}\right|, \\
& X_{i}^{t+1}=X_{i}^{t}+r_{1}{ }^{\star} \cos \left(r_{2}\right) \star\left|r_{3}{ }^{\star} p_{i}^{t}-X_{i}^{t}\right|,
\end{aligned}
$$

where $X_{i}^{t}$ is the position of the current solution in the $i^{t h} \mathrm{di}$ mension at the $t^{t h}$ iteration. $r_{1} / r_{2} / r_{3}$ are random numbers, $P_{i}$ is position of the destination point in the $i^{\text {th }}$ dimension, and $\|$ indicates the absolute value. These two equations are combined, to be used as follows:

$$
X_{i}^{t+1}=\left\{\begin{array}{lll}
X_{i}^{t}+r_{1}{ }^{\star} \sin \left(r_{2}\right) \star\left|r_{3}{ }^{\star} p_{i}^{t}-X_{i}^{t}\right| & \text { if } & r_{4}<0.5, \\
X_{i}^{t}+r_{1}{ }^{\star} \cos \left(r_{2}\right) \star\left|r_{3}{ }^{\star} p_{i}^{t}-X_{i}^{t}\right| & \text { if } & r_{4} \geq 0.5,
\end{array}\right.
$$

where $r_{4}$ is a random number in $[0,1]$. As the above equations show, there are four main parameters in SCA: $r_{1}, r_{2}$, $r_{3}$ and $r_{4}$. The parameter $r_{1}$ dictates the next position's region (or movement direction). The parameter $r_{2}$ defines how far the movement should be towards or away from the destination. The parameter $r_{3}$ brings a random weight for the destination in order to stochastically emphasize $\left(r_{3}>1\right)$ or deemphasize $\left(r_{3}<1\right)$ the effect of the destination in defining the distance. Finally, the parameter $r_{4}$ equally switches between the sine and cosine components in Equation (17). The SCA algorithm starts the optimization process by a set of random solutions. The algorithm then saves the best solution obtained so far, assigns it as the destination point, and updates other solutions with respect to it. Meanwhile, the ranges of sine and cosine functions are updated to emphasize exploitation of the search space as the iteration counter increases.

\subsection{The Ant Lion Optimizer}

Another novel nature-inspired algorithm so-called Ant Lion Optimizer (ALO) mimics the hunting mechanism of antlions in nature [34]. There are five main steps of hunting prey such as the random walk of ants, building traps, entrapment of ants in traps, catching prey, and re-building traps, all of which are implemented in this algorithm. Since ants move stochastically in nature when searching for food, a random walk is chosen for modelling ants' movement as follows:

$$
X(t)=\left[\begin{array}{ccc}
0 & \operatorname{cumsum}\left(2 r\left(t_{1}-1\right)\right) & \operatorname{cumsum}\left(2 r\left(t_{2}-1\right)\right) \\
\cdots & \left.\operatorname{cumsum}\left(2 r\left(t_{n}-1\right)\right)\right],
\end{array}\right.
$$

where cumsum calculates the cumulative sum, $\mathrm{n}$ is the maximum number of iterations, $t$ shows the step of ran- dom walk or iteration and $\mathrm{r}(\mathrm{t})$ is a stochastic function defined as follows:

$$
r(t)=\left\{\begin{array}{lll}
1 & \text { if } & \text { rand }>0.5 \\
0 & \text { if } & \text { rand } \leq 0.5
\end{array}\right.
$$

Here rand is a random number generated with uniform distribution in the interval $[0,1]$. The position of an ant is saved and utilized during optimization in the following matrix:

$$
M_{A N T}=\left[\begin{array}{cccc}
A_{1.1} & A_{1.2} & \ldots & A_{1 . d} \\
A_{2.1} & A_{2.2} & \ldots & A_{2 . d} \\
& \vdots & & \\
A_{n .1} & A_{n .2} & \ldots & A_{n . d}
\end{array}\right],
$$

Here $A_{i, j}$ shows the value of the $j^{\text {th }}$ variable (dimension) of the $i^{\text {th }}$ ant, $n$ is the number of ants, and $d$ is the number of variables. The position of an ant refers the parameters for a particular solution. A fitness (objective) function is utilized during optimization and the following matrix stores the fitness value of all ants as follows:

$$
M_{O A}=\left[\begin{array}{c}
f\left(\left[A_{1.1}, A_{1.2}, \ldots, A_{1 . d}\right]\right) \\
f\left(\left[A_{2.1}, A_{2.2}, \ldots, A_{2 . d}\right]\right) \\
\vdots \\
f\left(\left[A_{n .1}, A_{n .2}, \ldots, A_{n . d}\right]\right)
\end{array}\right],
$$

Here $f()$ is the objective function. In addition to ants, we assume the antlions are also hiding somewhere in the search space. In order to save their positions and fitness values the following matrices are utilized:

$$
\begin{gathered}
M_{\text {antlion }}=\left[\begin{array}{cccc}
A L_{1.1} & A L_{1.2} & \ldots & A L_{1 . d} \\
A L_{2.1} & A L_{2.2} & \ldots & A L_{2 . d} \\
\vdots & & \\
A L_{n .1} & A L_{n .2} & \ldots & A L_{n . d}
\end{array}\right] \\
M_{O A L}=\left[\begin{array}{c}
f\left(\left[A L_{1.1}, A L_{1.2}, \ldots, A L_{1 . d}\right]\right) \\
f\left(\left[A L_{2.1}, A L_{2.2}, \ldots, A L_{2 . d}\right]\right) \\
\vdots \\
f\left(\left[A L_{n .1}, A L_{n .2}, \ldots, A L_{n . d}\right]\right)
\end{array}\right] .
\end{gathered}
$$

Here $A L_{i, j}$ shows the $j^{\text {th }}$ dimension's value of the $i^{\text {th }}$ antlion, $n$ is the number of antlions, and $d$ is the number of variables (dimension) where $M_{O L}$ is the matrix for saving the fitness of each antlion $A L_{i, j}$.

\subsubsection{Random walks of ants}

Random walks are all based on equation (18). Ants update their positions $X_{i}^{t}$ with a random walk at every step 
of the optimization which is normalized using the following equation (min-max normalization) in order to keep it inside the search space:

$$
X_{i}^{t}=\frac{\left(x_{i}^{t}-a_{i}\right)^{\star}\left(d_{i}-c_{i}^{t}\right)}{\left(d_{i}^{t}-a_{i}\right)}+c_{i},
$$

where $a_{i}$ is the minimum of the random walk, $d_{i}$ is the maximum of the random walk, $c_{i}^{t}$ and $d_{i}^{t}$ indicates the minimum and the maximum, of $i^{\text {th }}$ variable at $t^{\text {th }}$ iteration respectively.

\subsubsection{Trapping in antlion's spits}

The ants walk in a hypersphere defined by the vectors $c$ and $d$, around a selected antlion, affected by antlions traps. In order to mathematically model this supposition, the following equations are proposed:

$$
\begin{aligned}
& c_{i}^{t}=\text { Antlion }_{j}^{t}+c^{t}, \\
& d_{i}^{t}=\text { Antlion }_{j}^{t}+d^{t},
\end{aligned}
$$

where $c^{t}$ is the minimum of all variables, $d^{t}$ indicates the vector including the maximum of all variables, $c_{i}^{t}$ and $d_{i}^{t}$ are the minimum and the maximum of all variables for $i^{\text {th }}$ ant. Antlion ${ }_{j}^{t}$ shows the position of the selected $j^{\text {th }}$ antlion at $t^{\text {th }}$ iteration.

\subsubsection{Building a trap}

In order to model the antlions hunting capability, a roulette wheel is employed for selecting antlions based on their fitness during optimization. Ants are assumed to be trapped in only one selected antlion. This mechanism gives higher chances to the fitter antlions for catching ants.

\subsubsection{Sliding ants towards antlions}

Once antlions realize that an ant is in the trap they shoot sand outwards from the center of the pit. This behaviour forces the escaping ant to slide backdown in the pit. For mathematically modelling this behavior, the radius of the ants's random walks hyper-sphere is decreased adaptively. The following equations are proposed in this regard:

$$
\begin{aligned}
& c^{t}=\frac{c^{t}}{I} \\
& d^{t}=\frac{d^{t}}{I} .
\end{aligned}
$$

In Equations (26) and (27), $\mathrm{I}$ is a ratio defined by $I=$ $10^{W^{\star} t / T}$, where $\mathrm{T}$ is the maximum number of iterations, and $W$ is a constant defined based on the current iteration. Basically, the constant $W$ can adjust the accuracy level of exploitation.

\subsubsection{Catching prey and re-building the pit}

For mimicking the final stage of the hunt process, it is assumed that catching prey occurs when ants become fitter (goes inside sand) than its corresponding antlion, which is required to update its position to the latest position of the hunted ant. This is defined by the following equation:

$$
\text { Antlion }_{j}^{t}=\text { Ant }_{i}^{t} \quad \text { if } \quad f\left(A n t_{j}^{t}\right)>f\left(\text { Antlion }_{j}^{t}\right) .
$$

\subsubsection{Elitism}

In this algorithm the best antlion obtained so far in each iteration is saved and considered an elite. Since the elite is the fittest antlion, it should be able to affect the movements of all the ants during each iteration. Therefore, it is assumed that every ant randomly walks around a selected antlion by the roulette wheel and the elite simultaneously, as follows:

$$
A n t_{i}^{t}=\frac{R_{A}^{t}+R_{E}^{t}}{2},
$$

where $A n t_{i}^{t}$ indicates the position of the $i^{t h}$ ant, $R_{A}^{t}$ and where $R_{E}^{t}$ are the random walk around the antlion and the elite, respectively. This are selected by the roulette wheel at the $t^{\text {th }}$ iteration.

\subsection{Grey wolf optimizer(GWO)}

GWO is a recent meta-heuristic optimizer inspired by grey wolves and proposed by [35]. It mimics the leadership hierarchy and the hunting mechanism of grey wolves in nature. As described in the literature, the GWO algorithm includes two mathematical models: encircling prey and hunting prey.

\subsubsection{The encircling behavior}

During hunting, the grey wolves encircle prey. The mathematical model is presented in the following equations:

$$
\vec{D}=\left|\vec{C} \overrightarrow{X_{p}}(t)-\vec{X}(t)\right|,
$$




$$
\vec{X}(t+1)=\overrightarrow{X_{p}}(t)-\vec{A} \vec{D},
$$

where $D$ is the distance, $X_{p}(t)$ is the position vector of the prey and $X(t)$ indicates the position of the grey wolf. Further, $t$ indicates the current iteration, and $\vec{A}$ and $\vec{C}$ are coefficient vectors.

\subsubsection{The hunting model}

The optimization algorithm is guided by $\alpha, \beta, \gamma$, the three best solutions obtained so far. the other search agents follow them and update their positions according to the best search agent. This is accomplished as follows:

$$
\begin{array}{lc}
\overrightarrow{D_{j}}=\left|\overrightarrow{C_{i}} \vec{X}_{j}(t)-\vec{X}(t)\right|, \quad j=\alpha, \beta, \gamma \\
\overrightarrow{X_{i}}=\overrightarrow{X_{j}}(t)-\overrightarrow{A_{i}} \overrightarrow{D_{j}}, & i=1,2,3 \\
\vec{X}(t+1)=\frac{\overrightarrow{X_{1}}+\overrightarrow{X_{2}}+\overrightarrow{X_{3}}}{3} . &
\end{array}
$$

\subsection{Whale optimizer algorithm (WOA)}

Like the GWO algorithm, the WOA is a recent metaheuristic optimization algorithm mimicking the hunting behavior of humpback whales [36]. The particularity of this approach is in the manner of the simulated hunting behavior which is made randomly, i.e. the best search agent to chase the prey and the use of a spiral to simulate bubblenet attacking mechanism of hump back whales. The philosophy of hunting can be described in three steps: encircling prey, bubble-net attacking method (exploitation phase), search for prey (exploration phase). To update the position of whales during optimization, to choose between either the shrinking encircling mechanism or the spiral model, a probability of $50 \%$ was assumed. The mathematical model is as follows:

$$
\vec{X}(t+1)=\left\{\begin{array}{ll}
\overrightarrow{X^{\star}(t)}-\vec{A} \vec{D}, & \text { if } \quad p<0.5 \\
\overrightarrow{D^{\prime}} \exp ^{b l} \cos (2 \pi l)+\overrightarrow{X^{\star}(t)}, & \text { if } \quad p \geq 0.5
\end{array},\right.
$$

where $p$ is a random number in [0 1]. The same approach based on the variation of the $\vec{A}$ vector can be utilized to search for prey (exploitation). Further, $\overrightarrow{D^{\prime}}$ indicates the distance of the $i^{\text {th }}$ whale to the prey ( best solution obtained so far $X^{\star}(t)$ ). Further, $b$ is a constant for defining the shape of the logarithmic spiral and $l$ is a random number in $[-1,1]$.

\section{Sliding mode control}

The principle of this type of control consists of bringing, whatever the initial conditions, the representative point of the evolution of the system on a hyper surface of the phase space. Representing a set of static relationships between the state variables. The sliding mode control generally includes two terms:

$$
U(t)=U_{e q}(t)+U_{n}(t)
$$

with $U_{e q}(t)$ : A continuous term, called equivalent control, and $U_{n}(t)$ : a discontinuous term, called switching control.

1. Equivalent control. The method proposed by Utkin [37], consists in admiting that in sliding mode everything happens as if the system was driven by a so-called equivalent command. The latter corresponds to the ideal sliding regime, for which not only the operating point remains on the surface but also for which the derivative of the surface function remains zero, $\dot{S}(t)=0$ (which is an invariant surface over time).

2. Switching control. The switching command requires the operating point to remain at the neighborhood of the surface. The main purpose of this command is to check the attractiveness conditions. The PID sliding surface for the sliding mode control can be indicated using the following equation:

$$
S(t)=k_{d} \dot{e}(t)+k_{p} e(t)+k_{i} \int_{0}^{t f} e(t) d(t),
$$

with: $K_{p}, K_{i}$ and $K_{d}$ mentioned as PID parameters. Further, $e(t)=q_{d}(t)-q(t) \rightarrow \dot{e}(t)=\dot{q}_{d}(t)-\dot{q}(t)$, where $q_{d}, q, \dot{q}_{d}$ and $\dot{q}$ are the desired and actual positions and speeds of the robot articulations, respectively. To calculate Ueq, it is necessary that $\dot{S}(t)=0$, so that we have the following:

$$
\begin{aligned}
& \rightarrow K_{p} \dot{e}(t)+k_{i} e(t)+k_{d} \ddot{e}(t)=0 \\
& \rightarrow \ddot{e}(t)=k_{d}^{-1}\left(k_{p} \dot{e}(t)+k_{i} e(t)\right)=\ddot{q}_{d}(t)-\ddot{q}(t)
\end{aligned}
$$

then

$$
\begin{aligned}
\ddot{q} & =M(q)^{-1}\left(U_{e q}(t)-H(q, \dot{q}) \dot{q}-G(q)-F(\dot{q})\right) \\
& =\ddot{q}(d)+K_{d}^{-1}\left(K_{p} \dot{e}(t)+K_{i} e(t)\right),
\end{aligned}
$$

$$
\begin{aligned}
U_{e q} & =M(q)\left(\ddot{q}_{d}+K_{d}^{-1}\left(K_{p} \dot{e}(t)+K_{i} e(t)\right)\right) \\
& +H(q, \dot{q}) \dot{q}+G(q)+F(\dot{q}) .
\end{aligned}
$$


To obtain the switching control signal Un for SMCPID controller, the Lyapunov function can be chosen as follows:

$$
\begin{aligned}
V & =\frac{1}{2} S^{T}(t) S(t) \\
\rightarrow & \dot{V}=S^{T}(t) \dot{S}(t)=S_{T}(t)\left[k_{p} \dot{e}(t)+K_{i} e(t)+K_{d} \ddot{e}(t)\right] \\
\dot{V} & =S^{T}(t)\left[K_{p} \dot{e}(t)+K_{i} e(t) K_{d}\left(\ddot{q}_{d}(t)\right.\right. \\
& -\left(M ( q ) ^ { - 1 } \left(U_{e q}(t)+U_{n}(t)-H(q, \dot{q}) \dot{q}-G(q)\right.\right. \\
& -F(\dot{q}))))] .
\end{aligned}
$$

Replacing Equation (36) in (37) we obtain the following:

$$
\begin{aligned}
\dot{V} & =S^{T}(t)\left[K_{p} \dot{e}(t)+K_{i} e(t)+K_{d}\left(\ddot{q}_{d}(t)\right.\right. \\
& -\left(M ( q ) ^ { - 1 } \left(M(q)\left(\ddot{q}_{d}^{-1}\left(K_{p} \dot{e}(t)+K_{i} e(t)\right)\right)\right.\right. \\
& +H(q, \dot{q}) \dot{q}+G(q)+F(\dot{q})+U_{n}(t) \\
& -H(q, \dot{q}) \dot{q}-G(q)-F(\dot{q}))))] .
\end{aligned}
$$

To ensure that $S^{T}(t) S(t)<0$, the switching control law can be taken as follows:

$$
U_{n}(t)=M(q) K_{d}^{-1} \lambda \operatorname{sign}(s(t))
$$

where, $\lambda$ is the switching control gain of the sliding mode controller, and the function $\operatorname{sign}(\mathrm{S}(\mathrm{t}))$ is defined as:

$$
\operatorname{Sign}(S(t))=\left\{\begin{array}{lll}
1 & \text { if } & S>0 \\
0 & \text { if } & S=0 \\
-1 & \text { if } & S<0 .
\end{array}\right.
$$

Finally, the PID-SMC torque with the demonstration of Lyapunov stability condition [25], presented as follows:

$$
\begin{aligned}
U(t) & =M(q)\left(\ddot{q}_{d}+K_{d}^{-1}\left(K_{p} \dot{e}(t)+K_{i} e(t)\right)\right) \\
& +H(q, \dot{q}) \dot{q}+G(q)+F(\dot{q}) \\
& +M(q) K_{d}^{-1} \lambda \operatorname{sign}\left(K_{p} e(t)\right. \\
& \left.+K_{i} \int_{0}^{t f} e(t) d t+K_{d} \dot{e}(t)\right) .
\end{aligned}
$$

\section{Simulation and Results}

The aim of this work is to optimize the parameters of SMC with PID surface for the trajectory control of a 2DOF robot manipulator based on minimizing ITAE and ISTE objective functions, mentioned as:

$$
J_{1}=I T A E=\int|e| t d t
$$

$$
J_{2}=I S T E=\int e^{2} t d t .
$$

Robot parameters have been taken, in application, are the masses of the two links $m_{1}=10 \mathrm{~kg}, m_{2}=5 \mathrm{~kg}$, and the lengths $l_{1}=1 \mathrm{~m}$ and $l_{2}=0.5 \mathrm{~m}$ with the gravity acceleration $\mathrm{g}=9.8 \mathrm{~m} / \mathrm{s}^{2}$. First we apply the algorithms described above to tune the SMC controller. The objective function values for different optimization algorithms obtained with ITAE and ISTE criteria have been shown in Table 1. It can be seen from Table 1 that with ITAE criterion, ALO exceeds others algorithms in terms of better cost function, which means that the ALO algorithm gives the best optimum. By cons in ISTE criterion, GWO and ALO optimizers provide close cost values considered as better costs obtained. The corresponding optimum parameters of SMC-PID are recapitulated in the Table 2.

A) SMC-PID optimization without disturbances.

Figure 1 shows the torque input applied to the first and the second articulations obtained so far by the different optimization algorithms. It is clear that the control signal obtained by the ALO and SCA optimization is very supple in comparison with those obtained by GWO and WOA algorithms. This is another selective criterion to confirm the superiority of the ALO algorithm over other algorithms. Figure 2 displays the trajectory tracking of 2DOF robot manipulator controlled by optimized SMC-PID controller with GWO, WOA, SCA and ALO algorithms respectively using ISTE criterion. We can see the good performance of the robot with different optimization methods. This prove the robustness of the evolutionary algorithms. The convergence history and fitness functions of these algorithms are depicted respectively in Figures 3 and 4. It's obviously clear from the Figure 3 that ALO and SCA have almost the same convergence curve, whereas GWO and WOA algorithms provide almost close convergence curve (Figure 3). While Figure 4 shows clearly the better convergence of ISTE obtained by ALO compared to others algorithms. The "sign" function, which is used in Equation (41) creates more chattering effect on the control torque. In practice, the chattering may cause damage to system components. Thence, in order to avoid the chattering effect, the sign function is replaced by the "tanh function"(hyperbolic tangent). The end effector position of the robot controlled by ALO-SMCPID controller in the plane XY was presented in Figures 5, 6 and 7 respectively. The resulting control input of different optimizers is depicted in Figure 8. 
Table 1: Cost function for the first and second articulation without disturbances.

\begin{tabular}{ccccc}
\hline \multirow{2}{*}{ Cost function } & \multicolumn{2}{c}{ ITAE } & \multicolumn{2}{c}{ ISTE } \\
\cline { 2 - 5 } & $1^{\text {st }}$ & $2^{\text {nd }}$ & $1^{\text {st }}$ & $2^{\text {nd }}$ \\
\hline SMC-PID-ALO & $9.669 \mathrm{e}-05$ & $3.896 \mathrm{e}-05$ & $1.149 \mathrm{e}-07$ & $6.858 \mathrm{e}-08$ \\
SMC-PID-SCA & $123.6 \mathrm{e}-05$ & $17170 \mathrm{e}-05$ & $1.302 \mathrm{e}-07$ & $1.602 \mathrm{e}-07$ \\
SMC-PID-WOA & $634.2 \mathrm{e}-05$ & $61.82 \mathrm{e}-05$ & $2.234 \mathrm{e}-07$ & $6.042 \mathrm{e}-08$ \\
SMC-PID-GWO & $82.2 \mathrm{e}-05$ & $300.9 \mathrm{e}-05$ & $2.095 \mathrm{e}-08$ & $8.118 \mathrm{e}-08$ \\
\hline
\end{tabular}
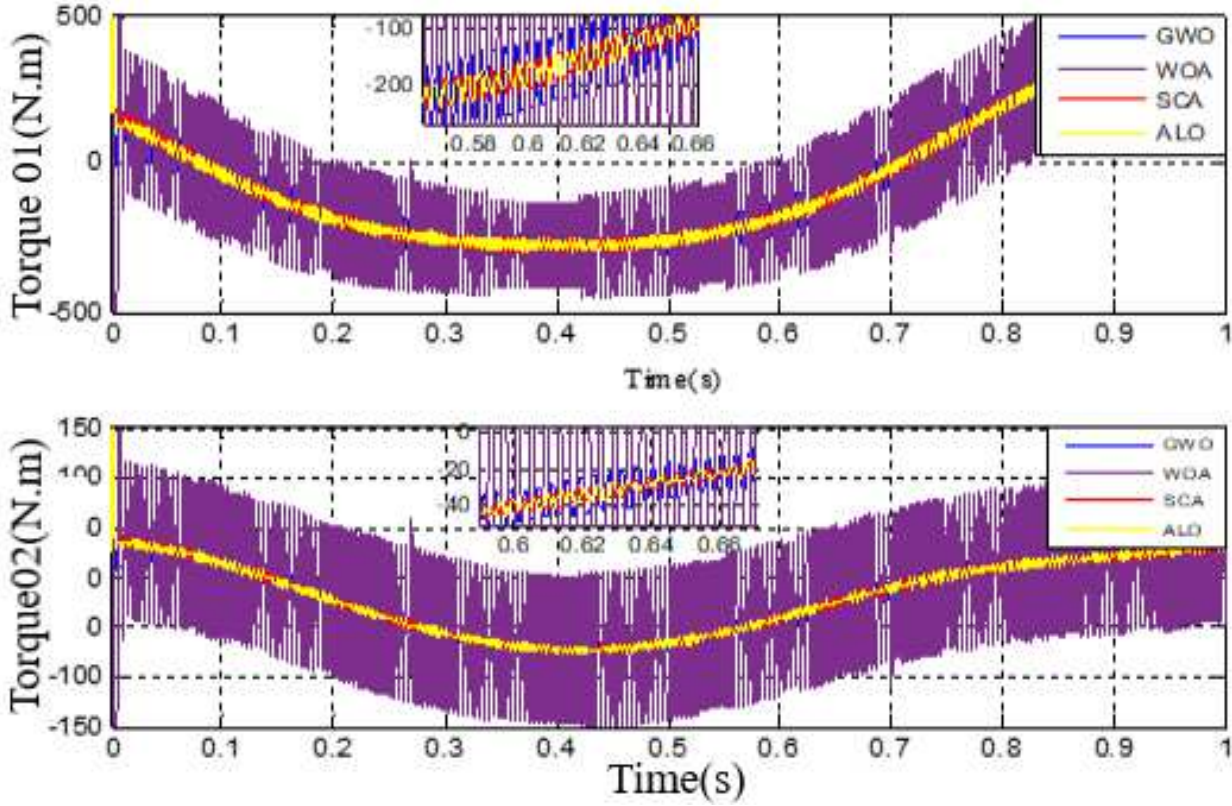

Figure 1: Control torque for first and second articulation of robot manipulator.
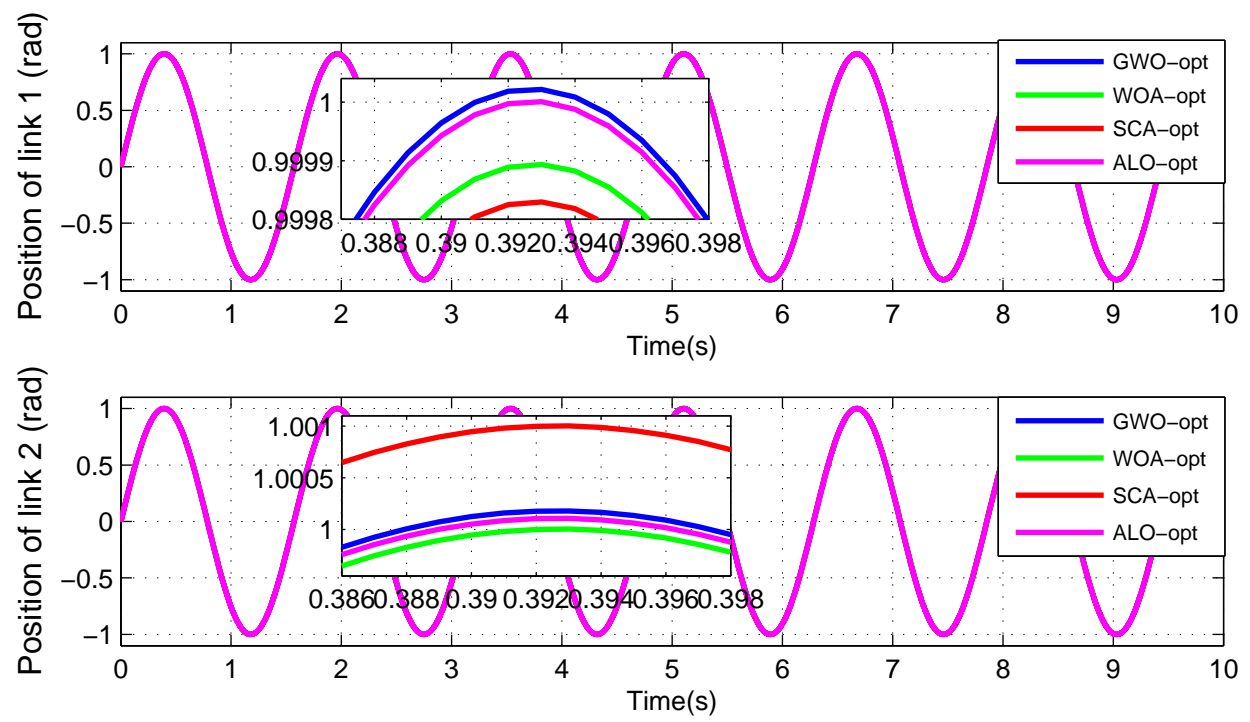

Figure 2: Trajectory tracking of link 1 and 2 with optimized SMC-PID controller without disturbances. 
Table 2: SMC-PID parametres for the first and second articulation without disturbances.

\begin{tabular}{ccccc}
\hline & $\begin{array}{c}\text { SMC-PID } \\
\text { ALO }\end{array}$ & $\begin{array}{c}\text { SMC-PID } \\
\text { SCA }\end{array}$ & $\begin{array}{c}\text { SMC-PID } \\
\text { WOA }\end{array}$ & $\begin{array}{c}\text { SMC-PID } \\
\text { GWO }\end{array}$ \\
\hline$K_{p 1}$ & 500 & 500 & 500 & 500 \\
$K_{i 1}$ & 52.46 & 1 & 55.31 & 49.89 \\
$K_{d 1}$ & 43.16 & 58.32 & 60.01 & 15.75 \\
$\lambda_{1}$ & 35.25 & 62.34 & 43.8 & 43.01 \\
$K_{p 2}$ & 500 & 500 & 500 & 603 \\
$K_{i 2}$ & 68.13 & 31.28 & 65.3 & 37 \\
$K_{d 2}$ & 30.43 & 53.55 & 1 & 46 \\
$\lambda_{2}$ & 41.49 & 14.02 & 45.65 & 48 \\
\hline
\end{tabular}

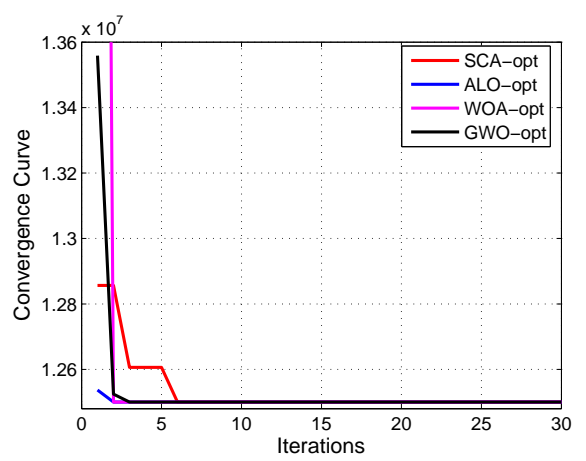

Figure 3: Convergence curve of different optimization algorithms.

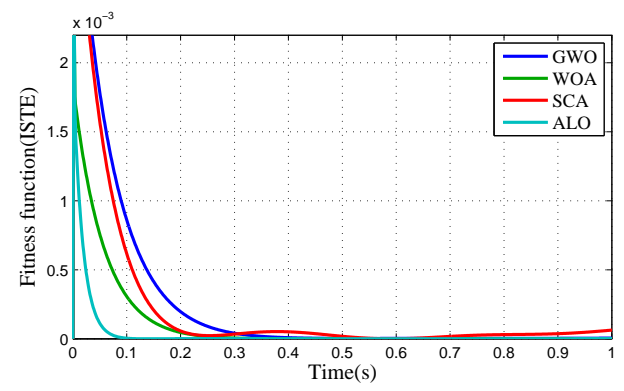

Figure 4: Convergence curves of ISTE criteria.

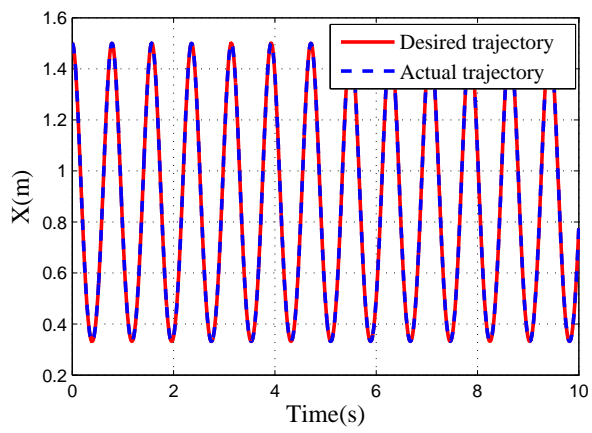

Figure 5: 2DOF robot end-effector $X$ position.

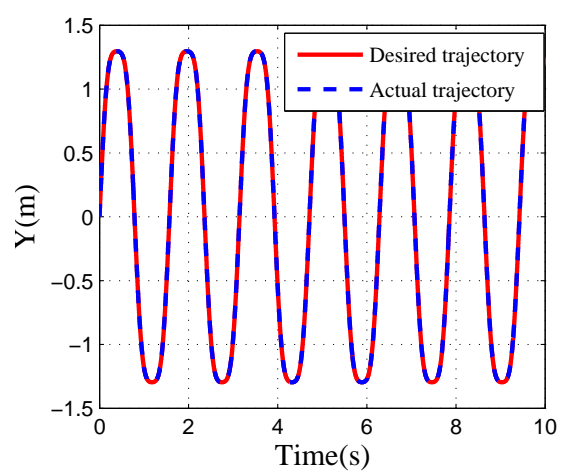

Figure 6: 2DOF robot end-effector Y position.

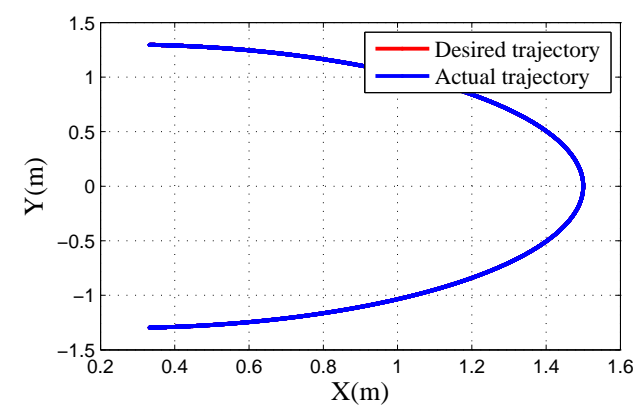

Figure 7: 2DOF robot end-effector position in XY plan controlled by ALO-SMC-PID controller.

B) SMC-PID optimization with torque disturbances. In the following, we proceed to the robustness analysis when the robot arm is subjected to external disturbance in the form of an additive signal to the control input. This disturbance is implemented with a white noise with different variances. Tables 3 to 6 list the SMC-PID parameters and the cost function values with external noise power. We can see from the robustness test of the SMC-PID that the cost function and the controller parameters obtained with GWO, WOA, SCA and ALO algorithms change relatively with the noise power. As can be seen (Figure 9), the ALO algorithm is the most robust to eliminate the effect of the torque disturbances. Consequently, the robot manipulator controlled by SMC-PID has a very good performance and becomes less sensitive to noise (with a small deviation from the reference trajectory occuring when the noise power increases). 

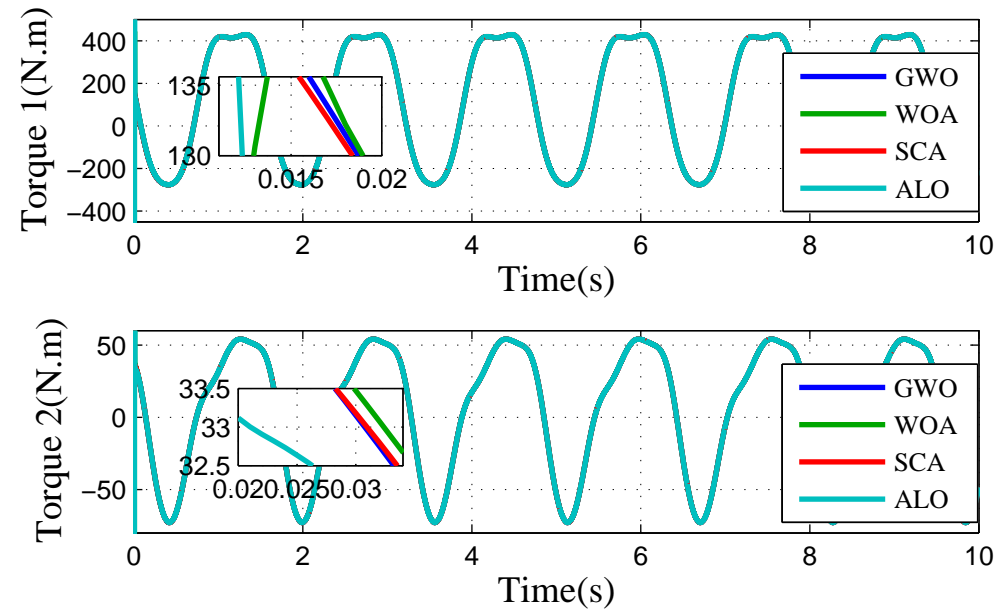

Figure 8: SMC-PID control input for first and second articulation of robot manipulator.
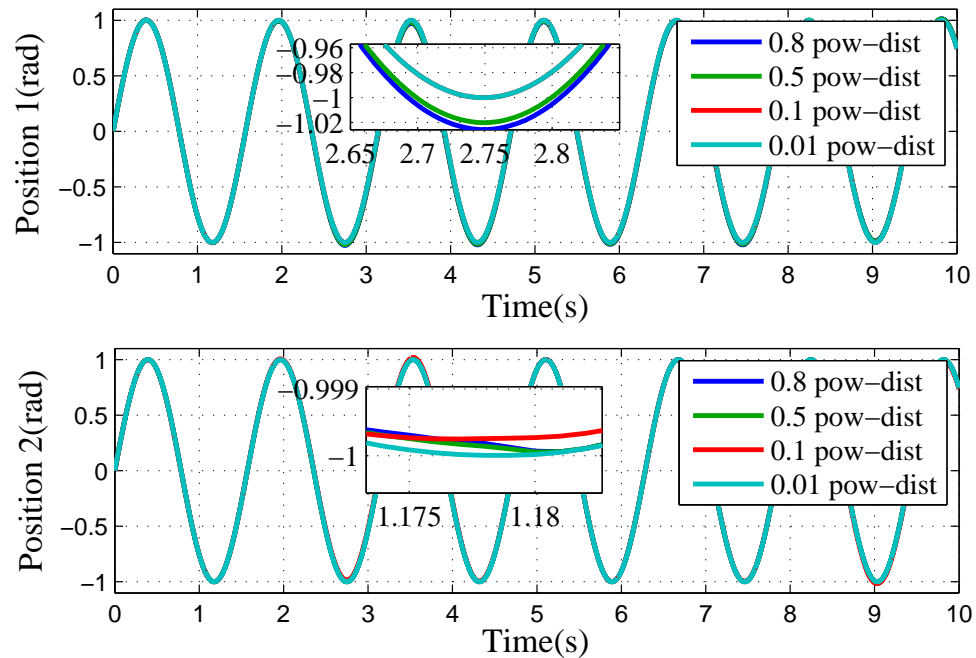

Figure 9: Tracking position of link 1 and 2 with SMC-PID optimized by ALO with disturbances.

Table 3: ISTE cost function in the presence of torque disturbances for GWO optimization.

\begin{tabular}{ccccc}
\hline$\delta$ & 0.01 & 0.1 & 0.5 & 0.8 \\
\hline$K_{p 1}$ & 500 & 500 & 500 & 500 \\
$K_{i 1}$ & 0 & 0 & 0 & 0 \\
$K_{d 1}$ & 52.39 & 92.83 & 17.33 & 37.34 \\
$\lambda_{1}$ & 30.19 & 92.62 & 70.73 & 100 \\
$K_{p 2}$ & 511 & 680 & 530 & 522 \\
$K_{i 2}$ & 4 & 5 & 34 & 28 \\
$K_{d 2}$ & 14 & 64 & 9 & 16 \\
$\lambda_{2}$ & 20 & 99 & 91 & 79 \\
ISTE1 & $2.489 \mathrm{e}-06$ & $2.752 \mathrm{e}-06$ & $1.552 \mathrm{e}-06$ & $4.393 \mathrm{e}-06$ \\
ISTE2 & $1.143 \mathrm{e}-05$ & 0.001413 & $4.034 \mathrm{e}-05$ & 0.001353 \\
ISTE1+ISTE2 & $1.3919 \mathrm{e}-05$ & 0.0014 & $4.1892 \mathrm{e}-05$ & 0.0014 \\
\hline
\end{tabular}


Table 4: ISTE cost function in the presence of torque disturbances for WOA optimization.

\begin{tabular}{ccccc}
\hline$\delta$ & 0.01 & 0.1 & 0.5 & 0.8 \\
\hline$K_{p 1}$ & 500 & 500 & 500 & 500 \\
$K_{i 1}$ & 0 & 0 & 0 & 0 \\
$K_{d 1}$ & 64.24 & 75.13 & 78.74 & 67.9 \\
$\lambda_{1}$ & 78.71 & 31.34 & 37.55 & 17.14 \\
$K_{p 2}$ & 500 & 000 & 500 & 500 \\
$K_{i 2}$ & 0 & 97.71 & 0 & 0 \\
$K_{d 2}$ & 11.16 & 27.45 & 30.44 & 36.93 \\
$\lambda_{2}$ & 70.53 & $2.119 \mathrm{e}-05$ & $28.22 \mathrm{e}-05$ & 67.23 \\
ISTE1 & $6.341 \mathrm{e}-07$ & 0.3255 & 0.005707 & $0.36 \mathrm{e}-04$ \\
ISTE2 & $1.074 \mathrm{e}-06$ & 0.3255 & 0.0060 & 0.0868 \\
ISTE1+ISTE2 & $1.7081 \mathrm{e}-06$ & & 0.0913 \\
\hline
\end{tabular}

Table 5: ISTE cost function in the presence of torque disturbances for SCA optimization.

\begin{tabular}{ccccc}
\hline$\delta$ & 0.01 & 0.1 & 0.5 & 0.8 \\
\hline$K_{p 1}$ & 500 & 500 & 500 & 500 \\
$K_{i 1}$ & 100 & 63.56 & 1 & 94.46 \\
$K_{d 1}$ & 89.48 & 100 & 11.61 & 55.81 \\
$\lambda_{1}$ & 72.73 & 38.02 & 19.49 & 45.07 \\
$K_{p 2}$ & 500 & 500 & 500 & 500 \\
$K_{i 2}$ & 20.69 & 100 & 50.8 & 89.79 \\
$K_{d 2}$ & 29.24 & 24.49 & 1 & 80.07 \\
$\lambda_{2}$ & 100 & $3.1 \mathrm{e}-05$ & 1.28 & 62.999 \\
ISTE1 & $1.568 \mathrm{e}-06$ & $5.834 \mathrm{e}-07$ & $0.61 \mathrm{e}-06$ & 0.0001073 \\
ISTE2 & $2.258 \mathrm{e}-06$ & $3.158 \mathrm{e}-5$ & 0.001134 & 1.875 \\
ISTE1+ISTE2 & $3.826 \mathrm{e}-6$ & & $1.2 \mathrm{e}-4$ & 1.8751 \\
\hline
\end{tabular}

Table 6: ISTE cost function in the presence of torque disturbances for ALO optimization.

\begin{tabular}{ccccc}
\hline$\delta$ & 0.01 & 0.1 & 0.5 & 0.8 \\
\hline$K_{p 1}$ & 500 & 500 & 500 & 500 \\
$K_{i 1}$ & 98.61 & 39.45 & 76.83 & 16.38 \\
$K_{d 1}$ & 72.98 & 25.57 & 16.19 & 36.23 \\
$\lambda_{1}$ & 41.75 & 35.38 & 62.87 & 1 \\
$K_{p 2}$ & 500 & 500 & 500 & 500 \\
$K_{i 2}$ & 23.64 & 1 & 39.96 & 1 \\
$K_{d 2}$ & 1 & 1 & 1 & 1 \\
$\lambda_{2}$ & 7.88 & 1 & 12.279 & 27.76 \\
ISTE1 & $2.633 \mathrm{e}-06$ & $2.191 \mathrm{e}-06$ & $1.76 \mathrm{e}-06$ & 0.0123 \\
ISTE2 & $2.093 \mathrm{e}-07$ & $2.164 \mathrm{e}-05$ & $6.484 \mathrm{e}-06$ & $4.224 \mathrm{e}-06$ \\
ISTE1+ISTE2 & $2.8423 \mathrm{e}-6$ & $4.355 \mathrm{e}-6$ & $8.244 \mathrm{e}-6$ & $1.2304 \mathrm{e}-2$ \\
\hline
\end{tabular}




\section{Conclusion}

In this paper, comparative study was carried out to test the effectiveness of the SMC with PID surface realized with new techniques of optimization called ALO, SCA, GWO and WOA algorithms. The ALO presents more robustness in trajectory tracking control of a 2 DOF robot manipulator in the presence of, or without, disturbances. Also, the signal input applied to the articulations was very supple in comparison with other optimization strategies, which is a very important factor to the safety of the actuators.

\section{References}

[1] Adhikary, N. Mahanta, C., Sliding mode control of position commanded robot manipulators, Control Engineering Practice, 2018, 183-198.

[2] Jung, S., Improvement of Tracking Control of a Sliding Mode Controller for Robot Manipulators by a Neural Network. International Journal of Control, Automation and Systems, 2018, 937-943.

[3] Yoo, D.S., A Comparison of Sliding Mode and Integral Sliding Mode Controls for Robot Manipulators, Transactions of the Korean Institute of Electrical Engineers, 2009.

[4] Liu, R. Li, S., Optimal integral sliding mode control scheme based on pseudo spectral method for robotic manipulators, International Journal of Control, 2014.

[5] ZHAO,Y. SHENG, Y. LIU, X., A Novel Finite Time Sliding Mode Control for Robotic Manipulators, IFAC Proceedings Volumes, 2014, 7336-7341.

[6] Azlan, N. Z. Osman, J. H.S., Proportional Integral Sliding Mode Control of Hydraulic Robot Manipulators with Chattering Elimination, First International Conference on Industrial and Information Systems, Sri Lanka, 2006.

[7] Das, M. Mahanta, C., Optimal second order sliding mode control for nonlinear uncertain systems, ISA Transactions, 2014, 11911198.

[8] Adelhedi, F. Jribi, A. Bouteraa, Y. Derbe, N., Adaptive Sliding Mode Control Design of a SCARA Robot Manipulator System Under Parametric Variations, Journal of Engineering Science and Technology Review, 2015, 117-123.

[9] Jiang, S. Zhao, J. Xie, F. Fu, J. Wang, X. Li, Z., A Novel Adaptive Sliding Mode Control for Manipulator with External Disturbance, 37th Chinese Control Conference CCC, 2008.

[10] Yi, S. Zhai, J., Adaptive second-order fast nonsingular terminal sliding mode control for robotic manipulators, ISA Transactions, 2019.

[11] Jing, C. Xu, H. Niu, X., Adaptive sliding mode disturbance rejection control with prescribed performance for robotic manipulators, ISA Transactions, 2019.

[12] Mirshekaran, M. Piltan, F. Esmaeili, Z. Khajeaian, T. Kazeminasab, M., Design Sliding Mode Modified Fuzzy Linear Controller with Application to Flexible Robot Manipulator, International Journal of Modern Education and Computer Science, 2013, 53-63.

[13] Tran, MD. Kang, HJ., Adaptive Fuzzy PID Sliding Mode Controller of Uncertain Robotic Manipulator, International Conference on In- telligent Computing, Intelligent Computing Theories and Methodologies ICIC, 2015, 92-103.

[14] Mohammad, A. Ehsan, SS., Sliding mode PID-controller design for robot manipulators by using fuzzy tuning approach, 27th Chinese Control Conference, 2008.

[15] Esfahani, HN. Azimirad, V., A New Fuzzy Sliding Mode Controller with PID Sliding Surface for Underwater Manipulators, International Journal of Mechatronics, Electrical and Computer Technology, 2013, 224-249.

[16] Kharabian, B. Bolandi, H. Ehyaei, A. F. Mousavi Mashhadi, S. K. Smailzadeh, S. M., Adaptive Tuning of Sliding Mode-PID Control in Free Floating Space Manipulator by Sliding Cloud Theory, American Journal of Mechanical and Industrial Engineering, 2017, 64-71.

[17] Medhaffar, H. Derbel, N. Damak, T., A decoupled fuzzy indirect adaptive sliding mode controller with application to robot manipulator, International journal of modeling, identification and control, 2006, 23-29.

[18] Qureshi, MS. Swarnkar, P. Gupta, S., Fuzzy PID Sliding Mode Control for Robotics: An Application to Surgical Robot, Recent Advances in Electrical and Electronic Engineering, 2019, 118-129.

[19] Firdaus, AR. Rahman, AS., Genetic Algorithm of Sliding Mode Control Design for Manipulator Robot, TELKOMNIKA, 2012, 645654.

[20] Long, Y. Du, Z]. Wang, WD. Dong, W., Robust Sliding Mode Control Based on GA Optimization and CMAC Compensation for Lower Limb Exoskeleton, Applied bionics and biomechanics, 2016, 13 pages.

[21] Zhou, T. Liang, XF., Position Sliding Mode Control of Manipulator Joint Based on Genetic Algorithm, Advanced Materials Research, 2014, 727-731.

[22] Rezapour, J. Sharifi, M. Nariman-Zadeh N., Application of fuzzy sliding mode control to robotic manipulator using multi-objective genetic algorithm, International Symposium on Innovations in Intelligent Systems and Applications, 2011, 455-459.

[23] Jalili-Kharaajoo, M. Rouhani, H., Fuzzy Sliding Mode Control of Robotic Manipulators Based on Genetic Algorithms, Advances in Artificial Intelligence, 2004, 892-900.

[24] Vijay, M. Jena, D., PSO based backstepping sliding mode controller and observer for robot manipulators, International Conference on Power, Instrumentation, Control and Computing PICC, 2018.

[25] Vijay, M. Jena, D., PSO based neuro fuzzy sliding mode control for a robot manipulator, Journal of Electrical Systems and Information Technology, 2017, 243-256.

[26] Khooban, MH. Soltanpour M., A particle swarm optimization approach for fuzzy sliding mode control for tracking the robot manipulator, Nonlinear Dynamics , 2013,74, 467-478.

[27] Ibrahim, AE. Gharghory S., Optimal Design of Super Twisting Control with PSO Algorithm for Robotic Manipulator, International Journal of Intelligent Systems and Applications in Robotics, 2018, 15 pages.

[28] Medjghou, A. Ghanai, M. Chafaa K., BBO optimization of an EKF for interval type-2 fuzzy sliding mode control, International Journal of Computational Intelligence Systems, 2018, 770 - 789.

[29] Jalali, A. Piltan, F. Keshtgar, M. Jalali M., Colonial Competitive Optimization Sliding Mode Controller with Application to Robot Manipulator, I.J. Intelligent Systems and Applications, 2013, 5056. 
[30] Oliveira, J. Oliveira, PM. Boaventura-Cunha, J. Pinho, T., Chaosbased grey wolf optimizer for higher order sliding mode position control of a robotic manipulator, Nonlinear Dynamics, 2017, 1353-1362.

[31] Mokeddem D. Draidi, H., Optimization of PID Sliding Surface Using Antlion Optimizer, International Symposium on Modelling and Implementation of Complex Systems MISC 2018, 133-145.

[32] Loucif, F. Kechida, S. Sebbagh, A., Whale optimizer algorithm to tune PID controller for the trajectory tracking control of robot manipulator, Journal of the Brazilian Society of Mechanical Sciences and Engineering, 2020, 42,1-14.
[33] Mirjalili, S., SCA A Sine Cosine Algorithm for solving optimization problems, Knowledge-Based Systems, 2016, 1-14.

[34] Mirjalili, S., The Ant Lion Optimizer, Advances in Engineering Software, 2015, 83, 80-98.

[35] Mirjalili, S. Mirjalili, SM. Lewis, A., Grey wolf optimizer, advance in engineering software, 2014, 46-61.

[36] Mirjalili, S. Lewis, A., The whale optimization algorithm, advance in engineering software, 2016,51-57.

[37] Utkin, VI., Slinding modes and their application in variable structure systems, In: MIR publisher Moscow, 1978. 